\title{
The cortical organization of language: distilling human connectome insights for supratentorial neurosurgery
}

\author{
Anujan Poologaindran, MSc, ${ }^{1,2}$ Stephen R. Lowe, MD, ${ }^{3}$ and Michael E. Sughrue, MD ${ }^{1,4}$ \\ 1Brain Mapping Unit, Department of Psychiatry, University of Cambridge; ${ }^{2}$ The Alan Turing Institute, London, United Kingdom; \\ ${ }^{3}$ Department of Neurosurgery, Medical University of South Carolina, Charleston, South Carolina; and ${ }^{4}$ Department of \\ Neurosurgery, Prince of Wales Private Hospital, Randwick, New South Wales, Australia
}

\begin{abstract}
Connectomics is the production and study of detailed "connection" maps within the nervous system. With unprecedented advances in imaging and high-performance computing, the construction of individualized connectomes for routine neurosurgical use is on the horizon. Multiple projects, including the Human Connectome Project (HCP), have unraveled new and exciting data describing the functional and structural connectivity of the brain. However, the abstraction from much of these data to clinical relevance remains elusive. In the context of preserving neurological function after supratentorial surgery, abstracting surgically salient points from the vast computational data in connectomics is of paramount importance. Herein, the authors discuss four interesting observations from the HCP data that have surgical relevance, with an emphasis on the cortical organization of language: 1) the existence of a motor speech area outside of Broca's area, 2) the eloquence of the frontal aslant tract, 3) the explanation of the medial frontal cognitive control networks, and 4) the establishment of the second ventral stream of language processing. From these connectome observations, the authors discuss the anatomical basis of their insights as well as relevant clinical applications. Together, these observations provide a firm platform for neurosurgeons to advance their knowledge of the cortical networks involved in language and to ultimately improve surgical outcomes. It is hoped that this report encourages neurosurgeons to explore new vistas in connectome-based neurosurgery.
\end{abstract}

https://thejns.org/doi/abs/10.3171/2020.5.JNS191281

KEYWORDS awake; glioma; resection; connectomics; language; surgical technique

$\mathrm{T}$ HE term "connectomics" refers to utilizing big data and computing approaches to assemble and analyze connections in the human brain. ${ }^{1}$ A major theoretical force that motivates connectomics stems from the idea that networks are fundamental to understanding the brain's structural and functional organization. ${ }^{2}$ While substantial breakthroughs have been made by basic neuroscientists, as neurosurgeons, we bring a rare and unique view on how to harness connectomics to determine precise anatomical relationships. ${ }^{3}$ The concept of operating on "connectomic anatomy" is attractive, with the clinical angle of abstracting from high dimensional data for surgically meaningful purposes.

To begin orienting neurosurgeons to connectomics, the senior author (M.E.S.) published an atlas detailing the anatomy of the human connectome with techniques such as coordinate-based meta-analysis. ${ }^{4,5}$ In the present review, we attempt to focus on what we believe to be, for neurosurgeons operating on the cerebral cortex, the most clear and clinically important ideas from connectomics. Specifically, we distill our observations from the Human Connectome Project (HCP) on the cortical organization of language and provide four key messages for neurosurgeons.

\section{Observation 1: Existence of a Speech Production Site in the Frontal Lobe Outside of Broca's Area}

Area 55b is one of the most intriguing brain areas discovered by the HCP. It is a small region that sits inside the precentral sulcus, immediately posterior to the middle

ABBREVIATIONS CEN = central executive network; DMN = default mode network; FAT = frontal aslant tract; fMRI = functional MRI; HCP = Human Connectome Project; IFOF = inferior fronto-occipital fasciculus; ILF = inferior longitudinal fasciculus; MTG = middle temporal gyrus; SLF = superior longitudinal fasciculus; SMA = supplementary motor area; WMT = white matter tract.

SUBMITTED May 7, 2019. ACCEPTED May 6, 2020.

INCLUDE WHEN CITING Published online July 31, 2020; DOI: 10.3171/2020.5.JNS191281. 

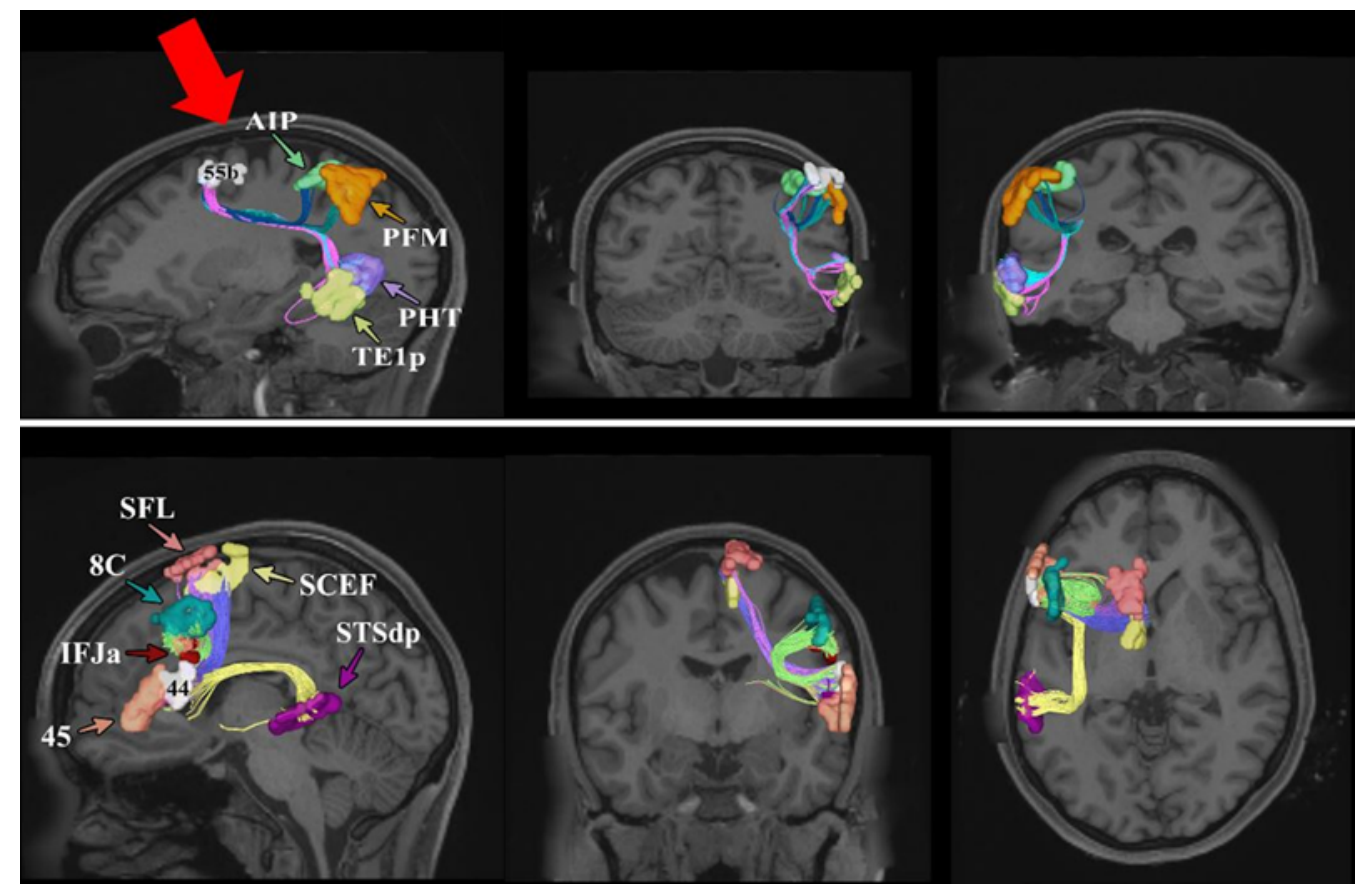

FIG. 1. The two speech pathways connecting to the semantic areas of the posterior left temporal lobe. Both the classic pathway (lower) and newer 55b-based pathway (upper) are represented. AIP = anterior intraparietal sulcus; IFJa = anterior inferior frontal cortex; PFM = Brodmann area 40 PFm complex; PHT = PHT complex of middle temporal gyrus; SCEF = supplementary and cingulate eye fields; SFL = superior frontal language area; $S T S d p=$ superior temporal sulcus dorsal posterior; TE1p = temporal area 1 posterior; $8 \mathrm{C}=$ Brodmann area 8 component $\mathrm{C}$.

frontal gyrus (Fig. 1). ${ }^{5,6}$ More specifically, area 55b sits between the frontal eye field (FEF) and premotor eye field (PEF) but is highly variable in its relationship with them; sometimes $55 \mathrm{~b}$ is bisected by a fused FEF/PEF. ${ }^{5}$ Taskbased functional MRI (fMRI) in the HCP data suggests that $55 \mathrm{~b}$ is activated in all tasks requiring language production, which is how it was distinguished from adjacent regions. Interestingly, our work also revealed that $55 \mathrm{~b}$ has a unique white matter tract (WMT) to the posterior tem-
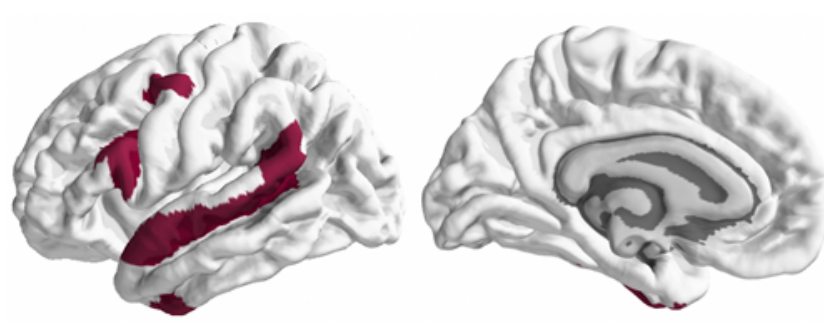

FIG. 2. Red and white indicate brain regions inside and outside, respectively, area 55b's modular community structure on the lateral (left) and medial (right) brain surface. In the HCP parcellation scheme, the clique is formed of 13 brain regions: area $55 \mathrm{~b}$, perisylvian language area (PSL), superior temporal visual area (STV), Brodmann area 44, inferior frontal junction anterior (IFJa), inferior frontal sulcus anterior (IFSa), superior temporal gyrus anterior (STGa), auditory complex 5 (A5), superior temporal sulcus dorsal anterior (STSda), superior temporal sulcus dorsal posterior (STSdp), area TF (TF), temporo-parieto-occipital junction 1 (TPOJ1), and area TG ventral (TGV). This community is overwhelmingly organized on the brain's lateral surface, highlighting the importance of determining an appropriate lateral entry zone during surgery (Video 1). poral language areas in the left hemisphere via the second division of the superior longitudinal fasciculus (SLF). Area 55b's eye field neighbors lack this unique connection. Recent electrocorticography work suggests that there is a distinct laryngeal motor cortex in this region, which is involved in speech production. ${ }^{6}$ Our own examination of independent component analysis (ICA) decomposition of resting-state data has shown that this region is usually found in ICA components of the left frontoparietal network. ${ }^{4,7,8}$ Using resting-state functional imaging of 337 HCP subjects, ${ }^{9}$ we illustrated that $55 \mathrm{~b}$ forms a modular community structure with the inferior frontal sulcus, perisylvian language area, superior temporal gyrus, superior temporal visual area, ventral temporal pole, and temporo-parietal-occipital junction (Fig. 2). Together, it is most likely that this newly discovered area 55 b plays an important integrative role in the cortical organization of language.

Is it clinically important to know that there is a small speech production region inside a sulcus that we were previously unaware of? Retrospective review of the senior author's own cases suggests that apraxia of speech can occur when area $55 \mathrm{~b}$ is transgressed..$^{10}$ We identified a patient with a tumor limited to this area who was left unable to speak despite otherwise intact language functions after surgery. ${ }^{10}$ The role of awake surgery in this region, especially microsurgical definition of sulcal depth for cortical mapping, is left to be defined but seems warranted in light of these data. Finally, understanding the functional significance of $55 \mathrm{~b}$ would be important for advancing a brain-machine interface for stroke and closed-loop deep 


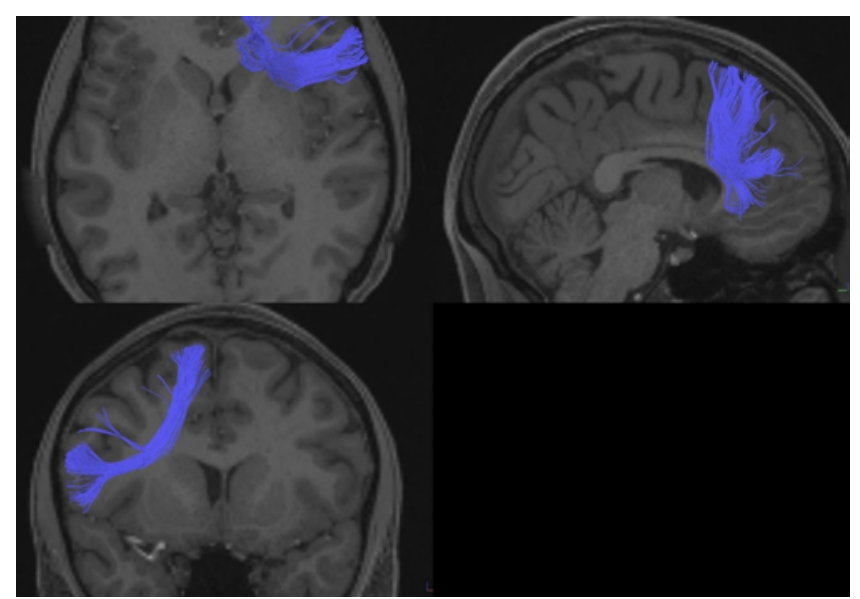

FIG. 3. Axial (upper left), sagittal (upper right), and coronal (lower left) diffusion spectrum imaging (DSI) tractography delineating the FAT, which connects the SMA to Broca's area and the ventral premotor areas, as well as the two parts of the salience network to each other.

brain stimulation ${ }^{11}$ for speech disorders such as spasmodic dysphonia, ${ }^{12}$ voice tremor, ${ }^{13}$ and stuttering. ${ }^{14}$

\section{Observation 2: The Frontal Aslant Tract Is Eloquent}

The frontal aslant tract (FAT) is a recently described WMT that runs orthogonal to the dominant direction of most tracts. ${ }^{15}$ Connectomic mapping by our group and others suggests that the FAT has two primary functions: 1) link the supplementary motor area (SMA) to the premotor areas and area 44, and 2) link the middle cingulate portions of the salience network to the anterior insular portions..$^{15}$ Thus, it is not surprising that intraoperative FAT stimulation leads to language arrest while tractography studies have implicated FAT degeneration in nonfluent primary progressive aphasias. ${ }^{16,17}$ In addition, recent data suggest that the FAT plays a key role in the genesis of postoperative SMA syndrome. Recent connectomic data provide a correlation between disruption of the "crossed" FAT and nonrecovery from postoperative SMA syndrome. ${ }^{15,18}$ The recovery of these deficits may be related to recruitment of the contralateral premotor area and SMA via a crossed FAT traversing the corpus callosum. ${ }^{18}$

Together, these findings suggest that the FAT appears to be a key component for systems of initiation and motor planning (Fig. 3). ${ }^{18-20}$ We have incorporated the FAT into our preoperative tractography for several years now and provide an illustrative case (Video 1).

VIDEO 1. Preoperative mapping and intraoperative approach to medial frontal glioma. The tumor is encroaching the SMA and FAT. The goal is to create a surgical plane that preserves the FAT (red) and the initiation axis (Fig. 4 left) while monitoring behavioral arrest with cortical mapping. Copyright Michael E. Sughrue. Published with permission. Click here to view.

Thus, it seems wise to avoid disconnecting the SMA from other motor planning areas. Future studies should employ prospective, longitudinal, serial connectome mapping of the FAT in SMA syndrome to further elucidate this unique neurosurgical problem.

\section{Observation 3: Connectome Explanation of the Medial Frontal Control Networks}

Classic models on the neurobiology of language have long posited that "bifrontal" injury leaves patients in an akinetic mute or abulic state. ${ }^{21}$ However, this model does not provide us with a sufficient map to avoid causing akinesia or abulia, especially in patients with bifrontal disease requiring surgery.

Recently, it has been demonstrated that at least three functional brain networks govern the transition between internal mentation and external actions. ${ }^{22,23}$ The default mode network (DMN), which is associated with passive unconstrained cognition, ${ }^{24,25}$ and the central executive network (CEN), which is associated with top-down cognitive
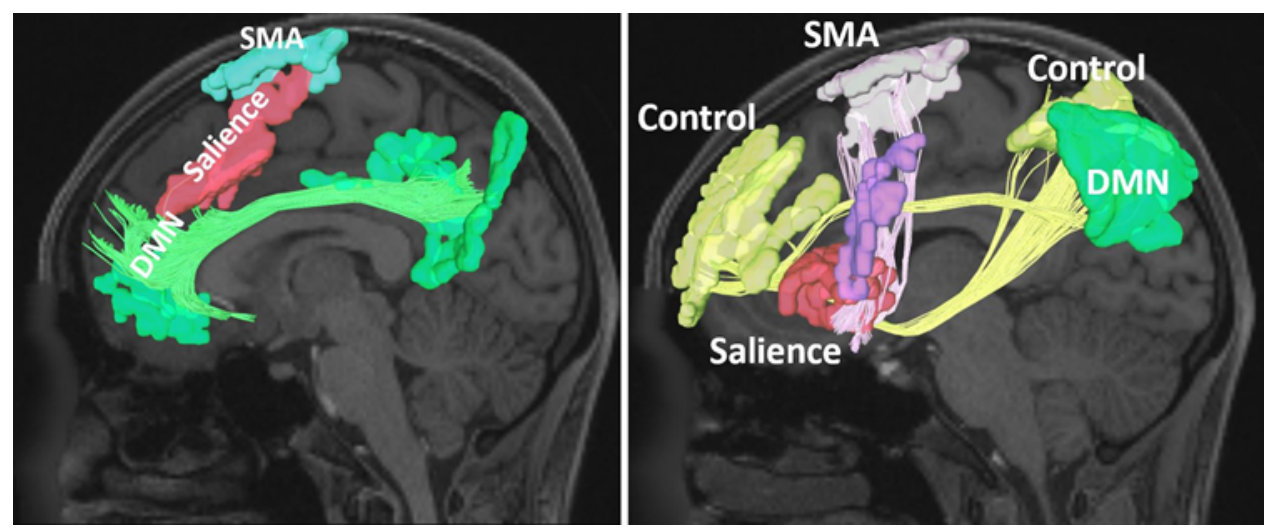

FIG. 4. Medial (left) and lateral (right) composite views of the multiple networks involved in the initiation axis. The DMN is in green, the salience network is in red, the SMA is in turquoise (left) and pink (right), the dorsal and ventral premotor areas are in white, and the control network is in yellow. Note that these networks are internally connected via white matter bundles (cingulum for the DMN, FAT for the salience and motor planning areas, and IFOF and SLF for the control network). A clinically important observation is that a band of networks on the medial surface involves the DMN, salience, and SMA areas; in fact, the SMA and salience networks seem to share a node. 
control,,$^{26}$ are functionally modulated in opposite directions (when one is active, generally the other is inactive). These two networks lie at the top of a cortical hierarchy of networks - this enables them to best process transmodal information unrelated to immediate stimulus input. ${ }^{27}$ The more recently described cingulo-opercular "salience" network is associated with facilitating the transition between DMN and CEN with stimulus orientating and task switching. ${ }^{1,28,51}$ Unsurprisingly, these networks are critical in several high-order cognitive tasks, and their dysfunctional connectivity is the basis of neurological and psychiatric illnesses. ${ }^{29,30}$

We became interested in this system when developing a cingulum-sparing technique during anterior butterfly glioma resections..$^{31}$ Specifically, we noticed that unilateral cingulate transgressions were not tolerated by a number of patients and that preserving the anterior cingulate improved outcomes by avoiding abulia or akinetic mutism. While unilateral cingulate damage is anecdotally known to be well tolerated in some instances, connectomics revealed that the DMN and salience network form a strip on the medial frontal lobe and anterior cingulate gyrus, which extends up to the SMA (Fig. 4). Thus, we hypothesize that maintaining the integrity of this strip, in addition to the cingulum portions of the DMN and the FAT connections to the SMA, is necessary to avoid causing initiation problems and akinesis in the majority of individuals. We recently provided direct evidence that sparing the cingulum during butterfly glioma surgery provides a near-zero rate of akinesis and abulia, while non-cingulum-sparing surgery results in a significantly higher rate of these deficits. ${ }^{31}$ Hence, a careful understanding of the cingulum's connectome anatomy and its association with the DMN is critical in the avoidance of these deficits following medial frontal lobe surgery (Video 1). In summary, given that unilateral cingulate damage can often be tolerated, we postulate that such transgressions do not necessarily induce abulia. Instead, surgical transgression of the DMN associated with the cingulum induces abulia-this viewpoint is supported by a detailed resting-state network study of an abulic stroke patient. ${ }^{32}$

\section{Observation 4: The Ventral Stream of Language Processing}

Unlike the previous three, our final observation eludes a simple and neat conclusion. Nevertheless, the HCP data provide valuable insights into this challenging but important topic on the second ventral stream of language processing: mapping "sound to meaning."

\section{Two New Components for Cortical Language Models}

Over the past several decades, it has been well established that the majority of individuals have two primary areas in the central core of language processing: Broca's area of the posterior inferior frontal gyrus and Wernicke's area of the posterior temporal lobe, both of which are mainly connected via the arcuate fasciculus. ${ }^{33}$ However, this classic model has failed to explain many clinical and scientific observations. Recent work in connectomics has started to unravel the true complexities of the language processing system. For example, apart from the wellknown addition of Geschwind's inferior parietal territory, ${ }^{34}$ the existence of the newly discovered area $55 \mathrm{~b}(\mathrm{ob}-$ servation 2 ) adds further complexity to the simple model. ${ }^{5}$ Furthermore, several components of the auditory network from the superior temporal gyrus also connect to area 44 (Fig. 5A) via the arcuate fasciculus that is independent of the WMT to the lateral temporal cortices. ${ }^{7}$ Thus, area 55b and auxiliary auditory WMTs are two additional aspects of the cortical organization of language that need to be accounted for in our models.

\section{The Elusive Anatomy of the Ventral Language Stream}

One of the most perplexing challenges surgeon-scientists face is to elucidate the exact anatomy of the second ventral stream of language production. ${ }^{35}$ We know this pathway exists for the following reasons: 1) left temporal lobectomies can leave patients with fluent speech despite complex higher-order disturbances such as those in verbal memory, ${ }^{36}$ 2) semantic dementia is a fluent but contentless aphasia typically seen in frontotemporal neurodegenerative disorders, ${ }^{37}$ and 3) direct intraoperative electrical stimulation of anteromedial temporal cortex elicits anomias. ${ }^{38}$ Thus, despite the ventral streams' existence, delineating its anatomy in language production is one of the most important challenges for our field. Given our frequent open surgical forays into the anterior temporal lobe and the development of more precise interventions in the temporal lobe, such as laser interstitial ablation, we are in a prime position to best solve this scientific puzzle.

In general, theories of the ventral stream anatomy of language production have revolved around three possible explanations (Fig. 6). 1) The inferior fronto-occipital fasciculus (IFOF) model: Given that direct stimulation of the IFOF at the temporoinsular stem induces anomias and that the IFOF clearly branches to area 45 , this theory posits that the IFOF carries semantic information from the visual and semantic systems to the inferior frontal language areas. $^{38-43}$ 2) The inferior longitudinal fasciculus (ILF) model: This model holds that the temporal pole plays a role in "semantic" function. Specifically, the ILF carries visual information to the temporal pole, which projects onward to area 45 via the uncinate fasciculus. ${ }^{38,44,45} 3$ ) The middle temporal gyrus (MTG) model: the semantic areas in the posterior MTG and superior temporal sulcus (STS) send fibers anteriorly to the temporal pole, which connects to area 45 via the uncinate.

In the following list, we summarize our own findings on this topic using the HCP data and suggest that two or even all three of these models may be simultaneously true to varying degrees. 1) Semantic tasks that sort objects based on meaning only activate areas classically associated with language, such as the STS and MTG ${ }^{46}$ In the senior author's parcellation of the HCP data (unpublished data), semantic tasks do not seem to activate the temporal pole-this is corroborated by independent fMRI studies. ${ }^{46,47}$

2) The IFOF does not connect to the semantic areas. In fact, it seems to mostly join with early visual areas such as V1-V4 (Fig. 5B) and dorsal stream areas involved in object position. ${ }^{40}$ We have never found any convincing 

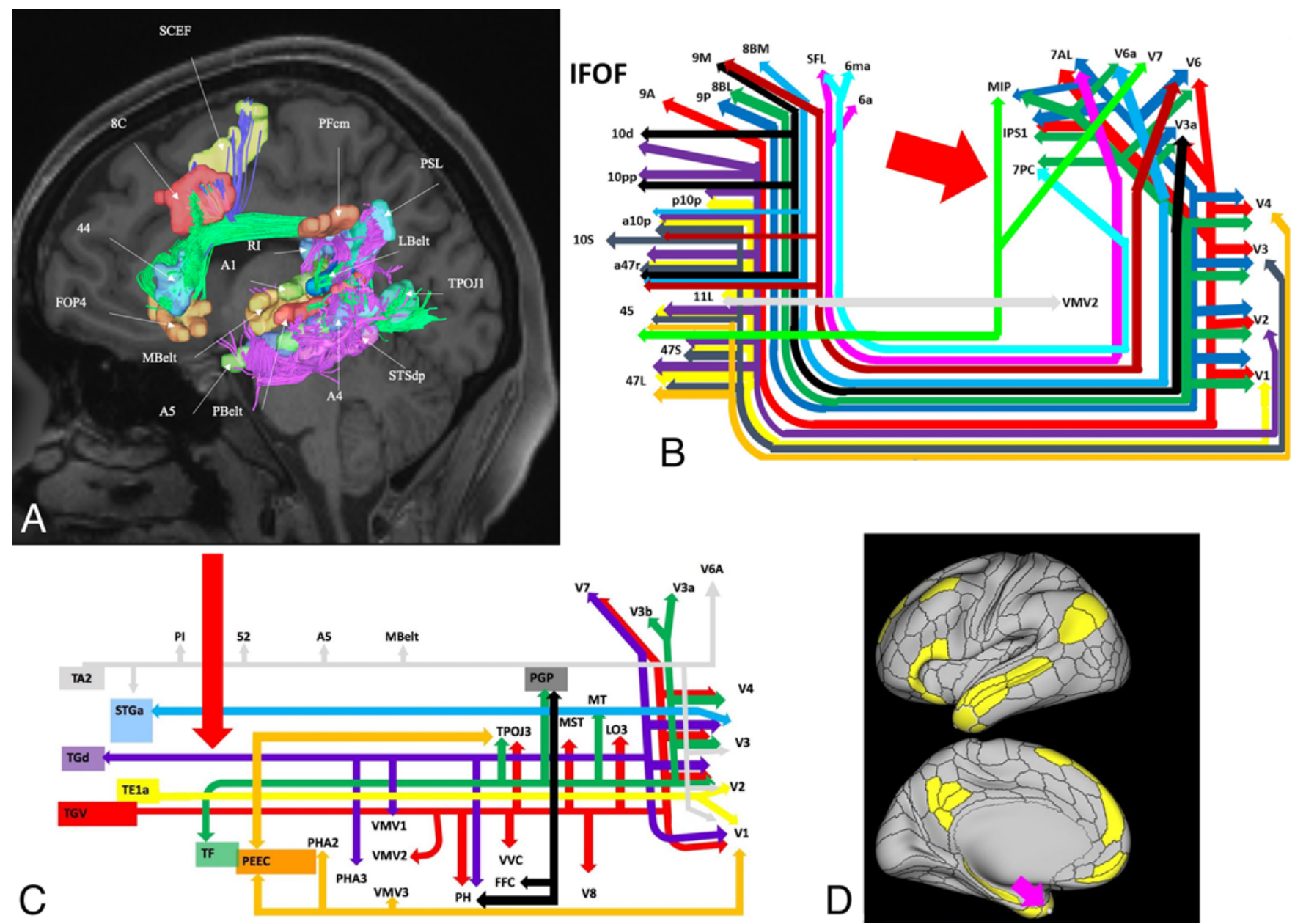

FIG. 5. Observations regarding the two-pathway model of language. A: In addition to the observations in Fig. 1, this figure demonstrates the auditory network, which has direct connections between early auditory areas and area 44, which are separate from the semantic area connections to area 44. B: A map of the IFOF connections from our previous work. A large red arrow highlights the direct connections to area 45, which are mostly parietal and dorsal to the visual stream. Also note the lack of connections to areas STSdp, TE1p, and PHT (which we have linked to semantic function). C: A map of the ILF connections from our previous work. A large red arrow highlights the direct connections to the temporopolar region temporal gyrus dorsal (TGd). Note again the lack of connections to areas STSdp, TE1p, and PHT. It does have some connections to the ventral visual stream (i.e., the "what" pathway) areas, such as PH, VMV1, and PHA3, but its most conspicuous connections are to early visual areas, such as V1-V3. D: Resting-state functional connectivity map of area TGd showing a band of functional connectivity along the MTG (yellow) which forms a bridge to semantic language area STSdp. Also note that the MTG is functionally connected to include language areas in the inferior frontal gyrus (IFG) area 45 (to which it is structurally linked via the uncinate fasciculus) and the three main components of the canonical DMN. Parcellations correspond to HCP cortical nomenclature: A1 = primary auditory cortex; $44=$ auditory 4 complex; A5 = auditory 5 complex; FFC = fusiform face complex; FOP4 = frontal opercular area 4; IPS1 = intraparietal sulcus area 1; LBelt = lateral belt complex; LO3 = area lateral occipital 3; MBelt = medial belt complex; MIP = medial intraparietal area; MST = medial superior temporal area; $\mathrm{MT}=$ middle temporal area; $\mathrm{PBelt}=$ parabelt complex; $\mathrm{PEEC}=$ perirhinal ectorhinal cortex; $\mathrm{PFcm}$ = Brodmann area 40 PFcm complex; PGP = area PGp near temporo-parieto-occipital junction; $\mathrm{PH}=$ anteroinferior lateral occipital lobe; PHA2 = parahippocampal area 2; PHA3 = parahippocampal area 3; PI = para-insular area; PSL = perisylvian language area; $\mathrm{RI}=$ retroinsular cortex; STGa = area STGa; TA2 = auditory association cortex area 2; TE1a = temporal area 1 anterior; TF = area TF of inferior temporal sulcus and gyrus; TGV = area TG ventral; TPOJ1 = temporo-parieto-occipital junction 1; TPOJ3 = temporoparieto-occipital junction 3; VMV1 = ventromedial visual area 1; VMV2 = ventromedial visual area 2; VMV3 = ventromedial visual area 3; VVC = ventral visual complex.

evidence that the IFOF contains fibers from the semantic language areas. In the specific case of area 45 , the connections seem to be to area V7, part of the dorsal visual stream (the "where" pathway) and the medial intraparietal area in the intraparietal sulcus, neither of which seems particularly useful for object recognition. ${ }^{40}$
3) The ILF connects to areas in the "what" pathway (Fig. 5C) or the ventral stream classically involved in object identification. ${ }^{45}$ However, there are a few problems with hypothesizing that the ILF carries information about object identity to the temporal pole. First, the temporal gyrus dorsal area, which is the majority of the temporal 


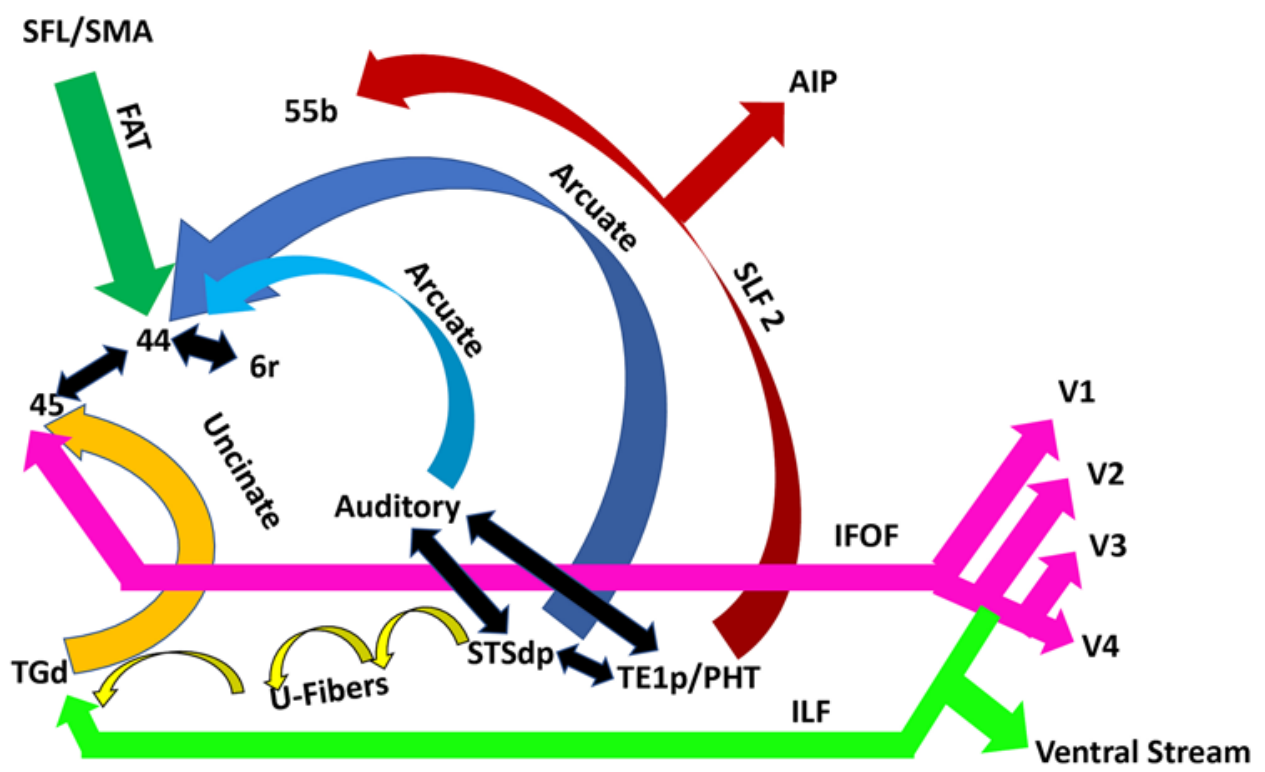

FIG. 6. Simplified composite line diagram demonstrating the various speech pathways. SLF $2=$ second division of the SLF.

pole in HCP nomenclature, does not appear to connect to many higher visual areas. Second, the classic visual recognition centers, the medial superior temporal area and middle temporal area, do not connect to the ILF; instead, they mainly connect to adjacent brain areas via the SLF.48 Finally, the ILF does not connect to the semantic areas. While these observations do not discredit the ILF model, they make it more difficult to accept.

4) The MTG model is functionally tenable: while the temporal pole does not activate during semantic tasks, it shows resting-state functional connectivity to areas that do. Importantly, there is a strip of areas along the MTG between the temporal pole area and semantic areas that is functionally connected and also seems to communicate with the areas of the DMN (Fig. 5D). Strikingly, using multiple auditory and visual task-based fMRI studies for each of 449 HCP subjects, Assem and colleagues recently demonstrated that the right MTG directly adjoins CEN and DMN parcels (temporal area 1 posterior and temporal area 1 middle, respectively), while the homologous left MTG adjoins two CEN parcels. ${ }^{49}$ This unique spatial proximity and hemispheric difference likely enables more efficient intra- and internetwork communication related to assigning the "meaning of sound." By integrating higher visual, auditory, and semantic language processing with self-referential introspective processes, the MTG model is functionally the most tenable to explain the second ventral stream for language production.

5) In addition to having a functional basis, the MTG model also has a structural basis. Interestingly, there is a small arcade of U-type fibers that parallels the MTG in an anterior-to-posterior direction, ${ }^{7}$ which likely mediates the aforementioned functional connectivity. Together, we believe that this band serves a role in processing semantic information to allow for memory retrieval and integration essential for naming tasks.

It is important to note that the strength of the MTG model does not necessarily exclude the validity of the other models. For example, in our experience, stimulation of the posterior temporoinsular junction induces language disturbance, and anatomically this would be hard to link to the uncinate alone. Presently, we consider the IFOF and ILF pathways organized as anterior-to-posterior pathways instead of posterior-to-anterior pathways. Specifically, it seems more plausible that the frontal and temporal regions use the IFOF and ILF to communicate with the early visual areas on how to process information and/or interpret information during memory retrieval. This is in contrast to the more traditional view that visual information is flowing forward in these pathways. Furthermore, our work suggests that the connections seem to be dominated with visual processing steps too early to meaningfully be carrying this information to a higher cognitive area such as $45 .{ }^{40,45}$ Early visual areas such as V1 are mainly concerned with processes such as line orientation and centersurround inhibition, ${ }^{50}$ which is not intuitively useful when naming objects. However, we know that visual processing is extremely reliant on top-down selection and modulation; thus, we should not exclude the possibility that the IFOF and/or ILF are important parts of this and that interrupting them could have consequences for language processing.

With these additional neuroscientific insights on the MTG model, how do we readily apply them to operative neurosurgery? While surgically avoiding eloquent fiber tracts such as the IFOF, ILF, and SLF and their corresponding eloquent cortices has long been practiced by neurosurgeons, avoiding the MTG is less emphasized given that it is often not possible, particularly in the case of temporal gliomas or anterior temporal lobectomy. Thus, this is a fundamental scientific and surgical problem the current generation of neurosurgeons faces and requires further attention and avant-garde approaches. Our group is actively tackling this problem with network neuroscience and deep learning. ${ }^{2}$ 


\section{Conclusions}

This work represents a brief and concise summary of several unique observations on the cortical organization of language gleaned from the HCP data and related investigations. While still a nascent field, connectomics has greatly contributed to our understanding of human language and other brain functions. The brain systems governing language can no longer be defined by the more simplistic models of the past but rather can be viewed as an orchestra of spatially distributed cortical areas, multiple WMTs, and whole-brain functional networks. Neurosurgeons are uniquely positioned to play a crucial role in establishing causal connectome-behavior relationships with millimetric and millisecond precision ${ }^{3,6}$ - an enviable opportunity not available to other scholars. The next step for our field will be to determine useful and reproducible connectome markers that can aid patient selection, operative neurosurgery, and postoperative rehabilitation. We provide a first step toward a deeper connectome-based insight into clinical problems, such as SMA syndrome following FAT injury or abulia following DMN disruption. We hope this paper provides a brief and concise overview of several unique observations on the connectome and stimulates neurosurgeons to explore new vistas in connectome-based neurosurgery.

\section{Acknowledgments}

Mr. Poologaindran is funded by a Turing Doctoral Scholarship from The Alan Turing Institute and the Natural Sciences and Engineering Research Council of Canada.

\section{References}

1. Glasser MF, Smith SM, Marcus DS, et al. The Human Connectome Project's neuroimaging approach. Nat Neurosci. 2016;19(9):1175-1187.

2. Bullmore E, Sporns O. Complex brain networks: graph theoretical analysis of structural and functional systems. Nat Rev Neurosci. 2009;10(3):186-198.

3. Poologaindran A, Suckling J, Sughrue ME. Elucidating the principles of brain network organization through neurosurgery. Letter. Neurosurgery. 2020;87(1):E80-E81.

4. Baker CM, Burks JD, Briggs RG, et al. A Connectomic Atlas of the Human Cerebrum-Chapter 1: Introduction, Methods, and Significance. Oper Neurosurg (Hagerstown). 2018; 15(suppl_1):S1-S9.

5. Baker CM, Burks JD, Briggs RG, et al. A Connectomic Atlas of the Human Cerebrum-Chapter 3: The Motor, Premotor, and Sensory Cortices. Oper Neurosurg (Hagerstown). 2018; 15(suppl_1):S75-S121.

6. Dichter BK, Breshears JD, Leonard MK, Chang EF. The control of vocal pitch in human laryngeal motor cortex. Cell. 2018;174(1):21-31.e9.

7. Baker CM, Burks JD, Briggs RG, et al. A Connectomic Atlas of the Human Cerebrum-Chapter 6: The Temporal Lobe. Oper Neurosurg (Hagerstown). 2018;15(suppl_1):S245-S294.

8. Baker CM, Burks JD, Briggs RG, et al. A Connectomic Atlas of the Human Cerebrum-Chapter 7: The Lateral Parietal Lobe. Oper Neurosurg (Hagerstown). 2018;15(suppl_1): S295-S349.

9. Ji JL, Spronk M, Kulkarni K, et al. Mapping the human brain's cortical-subcortical functional network organization. Neuroimage. 2019;185:35-57.

10. Chang EF, Kurteff G, Andrews JP, et al. Pure apraxia of speech after resection based in the posterior middle frontal gyrus. Neurosurgery. Published online February 25, 2020. doi:10.1093/neuros/nyaa002

11. Panov F, Levin E, de Hemptinne C, et al. Intraoperative electrocorticography for physiological research in movement disorders: principles and experience in 200 cases. J Neurosurg. 2017;126(1):122-131.

12. Poologaindran A, Ivanishvili Z, Morrison MD, et al. The effect of unilateral thalamic deep brain stimulation on the vocal dysfunction in a patient with spasmodic dysphonia: interrogating cerebellar and pallidal neural circuits. J Neurosurg. 2018;128(2):575-582.

13. Avecillas-Chasin JM, Poologaindran A, Morrison MD, et al. Unilateral thalamic deep brain stimulation for voice tremor. Stereotact Funct Neurosurg. 2018;96(6):392-399.

14. Craig-McQuaide A, Akram H, Zrinzo L, Tripoliti E. A review of brain circuitries involved in stuttering. Front Hum Neurosci. 2014;8:884.

15. Briggs RG, Conner AK, Rahimi M, et al. A Connectomic Atlas of the Human Cerebrum-Chapter 14: Tractographic Description of the Frontal Aslant Tract. Oper Neurosurg (Hagerstown). 2018;15(suppl_1):S444-S449.

16. Catani M, Mesulam MM, Jakobsen E, et al. A novel frontal pathway underlies verbal fluency in primary progressive aphasia. Brain. 2013;136(pt 8):2619-2628.

17. Kemerdere R, de Champfleur NM, Deverdun J, et al. Role of the left frontal aslant tract in stuttering: a brain stimulation and tractographic study. J Neurol. 2016;263(1):157-167.

18. Baker CM, Burks JD, Briggs RG, et al. The crossed frontal aslant tract: a possible pathway involved in the recovery of supplementary motor area syndrome. Brain Behav. 2018;8(3): e00926.

19. Kinoshita M, de Champfleur NM, Deverdun J, et al. Role of fronto-striatal tract and frontal aslant tract in movement and speech: an axonal mapping study. Brain Struct Funct. 2015; 220(6):3399-3412.

20. Selvanathan SK, Hammouche S, Salminen HJ, Jenkinson MD. Outcome and prognostic features in anaplastic ganglioglioma: analysis of cases from the SEER database. $\mathrm{J} \mathrm{Neu-}$ rooncol. 2011;105(3):539-545.

21. Toyoda K. Anterior cerebral artery and Heubner's artery territory infarction. Front Neurol Neurosci. 2012;30:120-122.

22. Baker CM, Burks JD, Briggs RG, et al. A Connectomic Atlas of the Human Cerebrum-Chapter 4: The Medial Frontal Lobe, Anterior Cingulate Gyrus, and Orbitofrontal Cortex. Oper Neurosurg (Hagerstown). 2018;15(suppl_1):S122-S174.

23. Briggs RG, Conner AK, Baker CM, et al. A Connectomic Atlas of the Human Cerebrum-Chapter 18: The Connectional Anatomy of Human Brain Networks. Oper Neurosurg (Hagerstown). 2018;15(suppl_1):S470-S480.

24. Greicius MD, Krasnow B, Reiss AL, Menon V. Functional connectivity in the resting brain: a network analysis of the default mode hypothesis. Proc Natl Acad Sci U S A. 2003; 100(1):253-258.

25. Raichle ME. The brain's default mode network. Annu Rev Neurosci. 2015;38:433-447.

26. Menon V, Uddin LQ. Saliency, switching, attention and control: a network model of insula function. Brain Struct Funct. 2010;214(5-6):655-667.

27. Margulies DS, Ghosh SS, Goulas A, et al. Situating the default-mode network along a principal gradient of macroscale cortical organization. Proc Natl Acad Sci U S A. 2016; 113(44):12574-12579.

28. Lamichhane B, Dhamala M. The salience network and its functional architecture in a perceptual decision: an effective connectivity study. Brain Connect. 2015;5(6):362-370.

29. Mak LE, Minuzzi L, MacQueen G, et al. The default mode network in healthy individuals: a systematic review and meta-analysis. Brain Connect. 2017;7(1):25-33. 
30. Otti A, Guendel H, Wohlschläger A, et al. Frequency shifts in the anterior default mode network and the salience network in chronic pain disorder. BMC Psychiatry. 2013;13:84.

31. Burks JD, Bonney PA, Conner AK, et al. A method for safely resecting anterior butterfly gliomas: the surgical anatomy of the default mode network and the relevance of its preservation. J Neurosurg. 2017;126(6):1795-1811.

32. Siegel JS, Snyder AZ, Metcalf NV, et al. The circuitry of abulia: insights from functional connectivity MRI. Neuroimage Clin. 2014;6:320-326.

33. Tremblay P, Dick AS. Broca and Wernicke are dead, or moving past the classic model of language neurobiology. Brain Lang. 2016;162:60-71.

34. Anderson JM, Gilmore R, Roper S, et al. Conduction aphasia and the arcuate fasciculus: a reexamination of the WernickeGeschwind model. Brain Lang. 1999;70(1):1-12.

35. Duffau H, Herbet G, Moritz-Gasser S. Toward a pluri-component, multimodal, and dynamic organization of the ventral semantic stream in humans: lessons from stimulation mapping in awake patients. Front Syst Neurosci. 2013;7:44.

36. Ojemann GA, Dodrill CB. Verbal memory deficits after left temporal lobectomy for epilepsy. Mechanism and intraoperative prediction. J Neurosurg. 1985;62(1):101-107.

37. Bang J, Spina S, Miller BL. Frontotemporal dementia. Lancet. 2015;386(10004):1672-1682.

38. Mandonnet E, Nouet A, Gatignol P, et al. Does the left inferior longitudinal fasciculus play a role in language? A brain stimulation study. Brain. 2007;130(pt 3):623-629.

39. Almairac F, Herbet G, Moritz-Gasser S, et al. The left inferior fronto-occipital fasciculus subserves language semantics: a multilevel lesion study. Brain Struct Funct. 2015;220(4): 1983-1995.

40. Conner AK, Briggs RG, Sali G, et al. A Connectomic Atlas of the Human Cerebrum-Chapter 13: Tractographic Description of the Inferior Fronto-Occipital Fasciculus. Oper Neurosurg (Hagerstown). 2018;15(suppl_1):S436-S443.

41. Khan OH, Herbet G, Moritz-Gasser S, Duffau H. The role of left inferior fronto-occipital fascicle in verbal perseveration: a brain electrostimulation mapping study. Brain Topogr. 2014;27(3):403-411.

42. Pilcher WH, Silbergeld DL, Berger MS, Ojemann GA. Intraoperative electrocorticography during tumor resection: impact on seizure outcome in patients with gangliogliomas. $J$ Neurosurg. 1993;78(6):891-902.

43. Sarubbo S, De Benedictis A, Maldonado IL, et al. Frontal terminations for the inferior fronto-occipital fascicle: anatomical dissection, DTI study and functional considerations on a multi-component bundle. Brain Struct Funct. 2013; 218(1):21-37.

44. Herbet G, Zemmoura I, Duffau H. Functional anatomy of the inferior longitudinal fasciculus: from historical reports to current hypotheses. Front Neuroanat. 2018;12:77.
45. Sali G, Briggs RG, Conner AK, et al. A Connectomic Atlas of the Human Cerebrum-Chapter 11: Tractographic Description of the Inferior Longitudinal Fasciculus. Oper Neurosurg (Hagerstown). 2018;15(suppl_1):S423-S428.

46. Duffau H, Moritz-Gasser S, Mandonnet E. A re-examination of neural basis of language processing: proposal of a dynamic hodotopical model from data provided by brain stimulation mapping during picture naming. Brain Lang. 2014;131:1-10.

47. Hickok G, Poeppel D. Dorsal and ventral streams: a framework for understanding aspects of the functional anatomy of language. Cognition. 2004;92(1-2):67-99.

48. Conner AK, Briggs RG, Rahimi M, et al. A Connectomic Atlas of the Human Cerebrum-Chapter 10: Tractographic Description of the Superior Longitudinal Fasciculus. Oper Neurosurg (Hagerstown). 2018;15(suppl_1):S407-S422.

49. Assem M, Glasser MF, Van Essen DC, Duncan J. A domaingeneral cognitive core defined in multimodally parcellated human cortex. Cereb Cortex. 2020;30(8):4361-4380.

50. Lanyon LJ, Barton JJ. Visual search and line bisection in hemianopia: computational modelling of cortical compensatory mechanisms and comparison with hemineglect. PLoS One. 2013;8(2):e54919.

51. Goulden N, Khusnulina A, Davis NJ, et al. The salience network is responsible for switching between the default mode network and the central executive network: replication from DCM. Neuroimage. 2014;99:180-190.

\section{Disclosures}

Dr. Sughrue has direct stock ownership in Omniscient Technologies.

\section{Author Contributions}

Conception and design: Sughrue. Acquisition of data: Sughrue. Analysis and interpretation of data: all authors. Drafting the article: all authors. Critically revising the article: all authors. Reviewed submitted version of manuscript: all authors. Approved the final version of the manuscript on behalf of all authors: Sughrue. Administrative/technical/material support: all authors. Study supervision: Sughrue.

\section{Supplemental Information Videos \\ Video 1. https://vimeo.com/417157946.}

\section{Correspondence}

Michael E. Sughrue: Prince of Wales Private Hospital, Randwick, NSW, Australia.sughruevs@gmail.com. 